\title{
El principio de insignificancia en el delito de hurto: Principales aspectos y su problemática
}

The principle of insignificance in the crime of theft: Main aspects and their problems

o princípio da insignificância no crime de furto: Aspectos principais e sua problemática

Le principe de l'insignifiance dans le crime de vol: les principaux aspects et leurs problèmes 盗窃罪中无关紧要的原则: 主要方面及其问题

\section{Tomás Camilletti ${ }^{1}$ \\ Universidad Nacional de La Plata - Argentina}

Revista Derechos en Acción ISSN 2525-1678/ e-ISSN 2525-1686

Año 5/N 16 Invierno 2020 (21 junio a 20 septiembre), 255-276

DOI: https://doi.org/10.24215/25251678e423

Recibido: 01/05/2020

Aprobado: 01/06/2020

Resumen: Este artículo nace en virtud de las problemáticas y consecuencias generadas en los delitos contra la propiedad cuando nos encontramos en presencia de cosas apoderadas de escaso o ínfimo valor. Asimismo, se pretende ofrecer al lector un repaso por todas las corrientes doctrinarias y jurisprudenciales de manera amplia, a los efectos de poder generar interrogantes en pos de capacitar a los operadores judiciales y aspirar a un sistema judicial más justo y armónico con los principios penales que rigen en nuestros tribunales. Respecto a ello, se intentarán analizar

\footnotetext{
Abogado graduado de la Facultad de Ciencias Jurídicas y Sociales (UNLP) en el año 2016. Docente adscripto en la materia Adaptación Profesional en Prácticas Penales. Actualmente cursando la Especialización en Derecho Penal de la Universidad de Buenos Aires. tomicamiIletti@gmail.com (ORCID: https://orcid.org/ 0000-0003-0895-6317).
} 
aquellas situaciones en la que, ante casos análogos propios del delito del hurto, la resolución del conflicto requiera un análisis pormenorizado de la circunstancia en particular y deje al descubierto la coyuntura generada con respecto al principio de insignificancia y la necesidad de aplicación de las penas. El eje de esta publicación no gira en torno a la conveniencia de aplicar una u otra manera de arribar a soluciones ante casos similares, sino que se provea de una serie de recursos y conocimientos que hagan tomar postura y generar argumentos suficientes, para así luego ello, poder repensar la finalidad de la aplicación de penas de corta duración, así como también la necesidad de realizar un cambio de paradigma en nuestro sistema penal

Palabras clave: Derecho Penal - Insignificancia - Principio de oportunidad - Delitos contra la propiedad.

\begin{abstract}
This article was born under the problems and consequences generated in property crimes when we are in the presence of seized things of little or very little value. Likewise, it is intended to offer the reader a review of all doctrinal and jurisprudential currents in a broad way, in order to be able to generate questions in order to train judicial operators and aspire to a more just and harmonious judicial system with the criminal principles that they rule in our courts. With regard to this, an attempt will be made to analyze those situations in which, in similar cases of the crime of theft, the resolution of the conflict requires a detailed analysis of the particular circumstance and reveals the situation generated with respect to the principle of insignificance and the need to apply penalties. The axis of this publication does not revolve around the advisability of applying one way or another to arrive at solutions to similar cases, but rather provides it with a series of resources and knowledge that make it take a stand and generate sufficient arguments, so that later, to be able to rethink the purpose of the application of short sentences, as well as the need to carry out a paradigm shift in our penal system.
\end{abstract}

Keywords: Criminal Law - Insignificance - Discretionary prosecution principle- crimes against property

Resumo: Este artigo nasce em virtude das problemáticas e consequências geradas nos crimes contra a propriedade quando estamos na presença de coisas apoderadas de pouco ou insignificante valor. Do mesmo modo, pretende oferecer ao leitor uma revisão por todas as correntes doutrinais 
e jurisprudenciais de forma ampla, a fim de poder gerar perguntas em procura de formar os operadores judiciários e aspirar a um sistema judicial mais justo e harmonioso com os princípios penais que regem em nossos tribunais. A este respeito, será tentado analisar as situações em que, perante casos semelhantes ao crime de furto, a resolução do conflito exige uma análise detalhada da circunstância particular e deixe ao descoberto a situação gerada em relação ao princípio da insignificância e à necessidade de aplicar penas. 0 eixo desta publicação não gira em torno da conveniência de aplicar uma ou outra forma para chegar a soluções para casos semelhantes, mas antes fornece uma série de recursos e conhecimentos que levarão à tomada de posição e à geração de argumentos suficientes, de modo a poder então repensar o propósito de aplicar penas de curta duração, assim como a necessidade de fazer uma mudança de paradigma no nosso sistema penal.

Palavras-chave: Direito Penal - Insignificância - Princípio de oportunidade - Crimes contra a propriedade.

Résumé: Cet article est né en vertu des problèmes et des conséquences engendrés par les délits contre les biens lorsque l'on se trouve en présence d'objets saisis de valeur faible ou négligeable. De même, il vise à offrir au lecteur une revue complète de tous les courants doctrinaux et jurisprudentiels, afin de pouvoir générer des questions afin de former les opérateurs judiciaires et aspirer à un système judiciaire plus juste et harmonieux avec les principes pénaux qui gouverner dans nos tribunaux. À cet égard, on tentera d'analyser les situations dans lesquelles, face à des cas analogues de crime de vol, la résolution du conflit nécessite une analyse détaillée de la circonstance particulière et révèle la situation générée au regard du principe d'insignifiance et de la besoin d'appliquer des sanctions. L'axe de cette publication ne tourne pas autour de la commodité d'appliquer d'une manière ou d'une autre pour parvenir à des solutions à des cas similaires, mais plutôt qu'elle fournit une série de ressources et de connaissances qui permettent de prendre position et de générer suffisamment d'arguments, pour le faire plus tard. , pour pouvoir repenser la finalité de l'application des peines de courte durée, ainsi que la nécessité de procéder à un changement de paradigme dans notre système pénal.

Mot-clés: Droit pénal Insignifiance Principe d'opportunité Crimes contre la propriété. 
摘要：当我们发现自己身处被扣押的价值不大或微不足道的东西的 情况下, 这是由于财产犯罪所产生的问题和后果而产生的. 同样, 其 目的是向读者提供对所有法学和法学流派的全面审查, 以便能够提 出问题，以培训司法操作人员并向往以司法原则为基础的更公正和 和谐的司法体系. 在我们的法庭上统治. 在这方面, 将尝试分析那些 面对类似盗窃罪的情况, 要解决冲突就需要对具体情况进行详细分 析, 并揭示在微不足道原则和不道德原则方面产生的情况. 需要进 行处罚. 本出版物的重点并不围绕采用一种方法或另一种方法来解 决类似案件的便利性，而是在于它提供了一系列资源和知识，使人 们有可能采取立场并产生足够的论据, 以便以后再做. 以便能够重 新考虑应用短期句子的目的, 以及在我们的刑法体系中进行范式转 换的需要.

关键字: 刑法, 无关紧要, 机会原则, 侵犯财产罪

\section{Introducción}

Esta presentación tiene sus orígenes en el relevante problema que se suscita en torno a los delitos contra la propiedad y la magnitud y/o límite de su objeto de tutela. Haciendo una reducción en el campo de investigación debido a la amplitud de problemáticas que traen aparejadas estos delitos, abordaremos en las próximas páginas particularmente el delito de hurto, tal como se tipifica en el artículo 162 del Código Penal Argentino.

Es moneda corriente observar en los tribunales nacionales y provinciales, un sinnúmero de causas que poseen como objeto la figura delictiva de referencia, donde muchas veces, el objeto apoderado ilegítimamente resulta de una manifiesta insignificancia respecto de todo lo que conlleva activar al aparato judicial cuando el mismo ingresa en la órbita penal y su eventual persecución a los fines de arribar a una resolución del conflicto.

Así pues, podemos observar que, con el correr de los años e inclusive en la actualidad, cuando el bien sustraído o apoderado posee una menor o insignificante cuantía, la divergencia de posturas y la disparidad de criterios que surgieron, han sido de distinta naturaleza a la hora de abordar estos problemas 
e interpretar la ulterior necesidad de activar todo el aparato punitivo estatal respecto a ellos.

Es por ello que en virtud de colaborar con la especificidad con la cual se debe tratar a la presente problemática, se tomará la figura del hurto simple prevista y reprimida en el artículo 162 del Código Penal Argentino, a los efectos de brindar un marco normativo y conceptual lo más claro posible, resultando este desarrollado como punto de partida para este trabajo.

El delito en estudio se encuentra previsto en el Libro Segundo, Título Sexto, Capítulo I del Código Penal de la República Argentina, en donde reza que "será reprimido con prisión de un mes a dos años, el que se apoderare ilegítimamente de cosa mueble, total o parcialmente ajena" (Código Penal Argentino, 1984, art. 162).

Es respecto a esta serie de conductas vedadas por el legislador que, sin pecar de sobreabundante y de hacer un análisis que se encuentre fuera del objeto de este trabajo, surgió a lo largo del tiempo, un problema respecto de la magnitud de la conducta delictiva en sí y la punibilidad de la misma ante casos particulares en donde la cosa total o parcialmente ajena y, por ende, dentro del patrimonio del sujeto pasivo como elemento objetivo típico, resultare insignificante o de mínimo valor. He allí donde comienza a relucir un cortocircuito con lo que se denominó principio de insignificancia o bagatela.

A modo introductorio, vemos cómo nos encontramos en presencia de una conducta delictiva reprimida donde el bien jurídico que se tutela es la propiedad, y en donde parte de la doctrina afirmó que lo genéricamente ofendido por el delito de hurto es la propiedad "como patrimonio, a cuyo contenido corresponde la tenencia de las cosas muebles, que dentro del género propiedad es lo específicamente ofendido" (Donna, 2007:26), y no en el sentido de propiedad o dominio como derecho real.

Por consiguiente, la figura del hurto, tendrá que consistir en el desapoderamiento de una cosa en poder material de 
otro, implicando ello una disminución de su patrimonio en su integridad (material), sin perjuicio de que ello no signifique un menoscabo en su integridad económica (Creus, 1998:391).

Por todo ello y los inconvenientes suscitados respecto a ello, a lo largo del trabajo se intentará realizar un breve repaso respecto soluciones procesalistas y dogmáticas ante situaciones que develen la ocurrencia de estos inconvenientes, intentando dotar la exposición de la mayor claridad argumental que sea posible y así poder brindarle al lector al final del mismo, un texto que le pueda significar de utilidad alguna a la hora de resolver casos análogos.

\section{Doctrina y jurisprudencia. Diversas interpretaciones}

Como bien señalamos con anterioridad, con el correr del tiempo diversas posturas doctrinarias y jurisprudenciales han abordado el problema que genera el principio en tratamiento y el acogimiento de la insignificancia en los delitos contra la propiedad, vale decir, considerando la irrelevancia a la hora de entender como típicas las conductas desplegadas en momentos de arribar a un decisorio de culpabilidad en el marco de los procesos penales.

Así pues, a continuación, se intentará abordar los principales aspectos de cada una de las posturas que se han manifestado al respecto, pretendiendo realizar una acabada delimitación de los objetos correspondientes a ellas.

\section{a. Principio de insignificancia. Su acogimiento doctrinario y jurisprudencial}

En primer lugar, es dable poner de manifiesto que el principio de insignificancia surge como principio aplicable a supuestos de injustos de poca importancia o valor (bagatelares), según el cual nos encontraríamos ante acciones o figuras penales atípicas, sin perjuicio que formalmente se adecuen a los elementos propios del tipo descripto, en virtud del menor 
contenido de injusto advertido en dicha conducta o cuando su reproche penal pudiese resultar desproporcionado en cada caso en concreto.

Surge de dicha interpretación que el principio de insignificancia tiene su origen en el Derecho Romano, donde el principio minimis non curat praetor ${ }^{2}$, el cual preveía su Digesto, pregonaba que "no debía ser oído el reclamante de cosa o cantidad mínima".

Ahora bien, respecto al abordaje de la teoría desplegada en derredor de la insignificancia podremos ver que existen dos niveles o perspectivas diversas desde dónde abordarlo como excluyente de responsabilidad penal o no punibilidad de la conducta típico-objetiva exteriorizada.

En primer término, nos encontraremos con un sector de la doctrina que sostiene un criterio que aborda el problema desde la dogmática penal la cual, prima facie, sostiene que las afectaciones insignificantes de ciertos bienes jurídicos no pueden constituir una ofensa de relevancia dentro de la faz objetiva de la tipicidad en las conductas exteriorizadas; mientras que por otro lado, cierto sector sostiene la no punibilidad de acciones bagatelares pero desde una faz procesal, en donde allí intervienen, juntamente con este principio, criterios de oportunidad en pos de velar por razones de celeridad, economía procesal y/o política criminal adecuada ajustadas a la actividad punitiva estatal.

Veamos:

\section{i. Perspectiva dogmática formal objetiva como fundamento de la atipicidad}

En primer término, establecidas las ideas principales en los apartados precedentemente desarrollados, huelga hacer mención que desde un primer momento, el Derecho Penal tiene por objeto la protección de ciertos bienes jurídicos, pudiendo

\footnotetext{
2 Real Academia Española (2020). Recuperado de https://dej.rae.es/lema/de-minimis-noncurat-praetor
} 
observar que dicha premisa posee íntima correlación con el principio de lesividad, en donde tal máxima actúa como limitativa del poder punitivo estatal.

Según Zaffaroni (2014:111), el discurso legitimante del punitivismo reaccionario racionalizó el hecho que "ante un bien jurídico lesionado, estaríamos en presencia de un delito y por lo tanto cabría una imposición de pena", y es por ello que con posterioridad, dicha perspectiva tuvo un giro de radical y pasó de entenderse al bien jurídico lesionado como bien jurídico tutelado.

En otro orden de ideas, el profesor Roxin (1997:55-56) sostuvo en consonancia con ello que "un concepto de bien jurídico vinculante político-criminalmente, solo se puede derivar de los cometidos, plasmados en la ley fundamental, de nuestro Estado de Derecho basado en la libertad del individuo, a través de los cuales se marcan sus límites a la potestad punitiva del Estado", por lo que "los bienes jurídicos son circunstancias dadas o finalidades que son útiles para el individuo y su libre desarrollo, en el marco de un sistema social estructurado sobre la base de esa concepción de los fines o para el funcionamiento del propio sistema".

Ahora bien, sentado el piso mínimo supra descripto, encontramos al momento, y de forma minoritaria, precedentes jurisprudenciales armónicos con posturas doctrinarias las cuales sostienen la relevancia y pertinencia de la insignificancia en estas conductas -de antemano- típicas.

El Dr. Raúl E. Zaffaroni desarrolló su clásica y reconocida teoría de la tipicidad y atipicidad conglobante.

Sobre la base de lo expuesto, el actual juez de la Corte Interamericana de Derechos Humanos sostiene que, en el análisis del tipo objetivo, deben distinguirse por un lado una función sistémica, la cual permite afirmar la existencia de un espacio problemático, y por otro, una función conglobante la cual permitirá averiguar la existencia de conflictividad. Al respecto refiere que "no basta con la consideración aislada del tipo, sino 
que debemos valernos de la misma fórmula legal considerada como parte de todo un conjunto orgánico normativo (el orden normativo), es decir, conglobada con todo el resto de normas vigentes" (Zaffaroni, 2014:356-357).

Afirma Zaffaroni que:

La consideración conglobada de las normas que se deducen de los tipos penales, es decir, su análisis conjunto, muestra que tienden en general, como dato de menor irracionalidad, a prohibir conductas que provocan conflictos de cierta gravedad. No se trata de sólo una manifestación del principio de última ratio, sino del propio principio republicano, del que se deriva directamente el principio de proporcionalidad, como demanda de cierta relación entre la lesión al bien jurídico y la punición (Zaffaroni, 2014:472).

Haciendo una interpretación conforme a los principios referenciados ante la ocurrencia de estos eventos, los mismos deberían hacer las veces de valladar infranqueable para los jueces aplicadores de la norma, los cuales deberán apartarse de todo tipo de soluciones de índole dogmática que disten ampliamente de un análisis concreto según las circunstancias particulares de cada caso.

Planteado ello, no resultará del todo aplicable la situación que, ante un hecho determinado como subsumido en una figura típico-penalmente relevante, la misma no sea analizada a la luz de una concreta lesividad, contemplando su significancia y la relevancia social que dicha conducta desplegó, así como también la racionalidad y proporcionalidad de la respuesta punitiva que pudiese caberle. Ello así toda vez que, como se ha explicado con anterioridad y tal como lo podremos observar en alguno de los precedentes que abordaré a continuación, el Derecho Penal a través de la tipificación de ciertas conductas, no pretende proteger todos los bienes jurídicos (lesionados), sino que intentará tutelarlos a efectos de su protección integral y abacartiva, lo cual nos lleva a concluir que podríamos encontrarnos frente a situaciones en las cuales la afectación, no supere el estándar 
previsto por la norma y por ello quedaran excluidos del tipo. Situación tal que se verifica con las ínfimas lesiones en este caso al derecho de propiedad.

Es por ello que en este orden de ideas, la insignificancia de la afectación excluirá la tipicidad siempre y cuando la misma sea interpretada desde una visión conglobada de la norma. Así pues, la insignificancia solo podrá surgir a la luz de una función general que le dé sentido al orden normativo y, consecuentemente, a la norma en particular, la cual nos indique la exclusión de dichos supuestos en el ámbito de la prohibición, lo cual sería de difícil concreción, si la misma se estableciera a la luz de una consideración aislada (Zaffaroni, 1981:555).

Ahora bien, vasta jurisprudencia ha sostenido dicha postura con el correr del tiempo, la cual nos parece importante destacar brevemente, tenga o no en la actualidad un acogimiento mayoritario en la doctrina, a los efectos de generar una mayor claridad de las tesituras anteriormente establecidas:

En primer término, estimamos útil reseñar un fallo de la Sala II de la Cámara Nacional de Casación en lo Criminal y Correccional, de fecha 10 de julio del año 2017, en donde se trató un recurso interpuesto por la defensa, donde una persona había sido descubierta por personal de seguridad en una perfumería, sustrayendo dos desodorantes y un jabón, y en donde el sindicado fue condenado en primera instancia por el delito de hurto en grado de tentativa ${ }^{3}$ a quince días de prisión.

Abierto a debate y admitido el remedio procesal interpuesto, la Cámara de Casación Nacional encabezó su resolutorio con el voto del Dr. Daniel Morín, el cual realizó un amplio desarrollo de la problemática instaurada respecto los hechos "bagatelares" y su abordaje doctrinario y jurisprudencial en la actualidad, en donde luego por unanimidad (Dres. Morín, Niño y Sarrabayrouse), se hizo lugar al recurso interpuesto, se casó

3 Cámara Nacional de Casación en Lo Criminal y Correccional - Sala 2, “Cutule, Pablo Alejandro s/hurto en tentativa", 10.07.2017. Recuperada de: http://www.pensamientopenal. com.ar/system/files/2017/11/fallos45935.pdf 
la resolución del Juzgado Nacional en lo Correccional Nro. 6 y se absolvió al imputado por el delito acusado.

La Sala II arribó a la conclusión que la acción imputada al encartado en autos resultó atípica, visto que el hurto de dos desodorantes de pie y un jabón encarna un supuesto que ofrece un mínimo disvalor objetivo del acto donde, por ejemplo, en un primer momento no fueron contempladas las circunstancias personales del imputado (tal como lo fueran su desempleo, su nivel de instrucción elemental y que el mismo es una persona en situación de vulnerabilidad como portador de VIH), así como tampoco se habían contemplado las particularidades del hecho, habiéndose hecho cargo el encausado de la imputación y contribuido a su esclarecimiento. A su vez, se señala que respecto el caso en particular, la sustracción se efectuó sin violencia y los elementos habían sido recuperados por el establecimiento, no merituándose siquiera el daño concreto en relación a la víctima. Así pues, la Cámara entendió que, en el caso, "el hurto de dos desodorantes y un jabón mal pudieron lesionar significativamente el patrimonio de la empresa "Farmacity".

A su vez, se puede extraer del resolutorio (según el voto del Dr. Niño) que, la conducta resulta atípica en cuanto el principio de racionalidad de los actos de gobierno, impuesto por la forma republicana (cfr. art. 1 de la Constitución Nacional del año 1853):

Obliga al operador judicial del subsistema penal a ejercer un control de tipicidad conglobante o sistemática, tendiente a recortar el haz de proyección de la tipicidad legal en aquellos casos en que no haya afectación del bien jurídico, o esta no fuera significativa (con cita de: Zaffaroni, Eugenio Raúl; Alagia, Alejandro; Slokar, Alejandro: Derecho Penal. Parte General, Ediar, Bs. As., 2000, p. 485.).

En síntesis, el Dr. Sarrabayrouse arriba a la conclusión que:

Si la función del Derecho penal es la protección subsidiaria de bienes jurídicos y que debe ser la última ratio del sistema, la conducta desplegada por [el imputado] no 
afectó el patrimonio de "Farmacity" pues no sólo los productos que intentó llevarse son de un valor económico escaso sino que también fueron recuperados.

Ahora bien, además de la doctrina legal reseñada, otros precedentes han abordado las situaciones problemáticas en tratamiento, como por ejemplo la Sala VI de la Cámara Nacional de Apelaciones en lo Criminal y Correccional, en donde el Dr. Bunge Campos estableció que no toda lesión al bien jurídico "propiedad" configura la afectación típica requerida ${ }^{4}$.

Sentando postura respecto a dicha máxima, se ha expresado que se debe recordar que:

El patrimonio no es un elemento abstracto, sino un atributo de la personalidad, como tal no debe juzgarse en forma aislada, sino en relación con su titular. Lo que para uno es una afectación nimia e insignificante, para otro puede ser una afectación trascendente. Por ello, el análisis de la afectación al bien jurídico no puede hacerse en forma abstracta o meramente formal. Desde el punto de vista de la teoría del delito, la afectación del bien jurídico cumple una función limitante de la tipicidad, no integrándola, de modo tal que una lesión insignificante, resultaría, por ende atípica al no revestir entidad suficiente para demandar la intervención del Estado. En este punto debemos necesariamente recordar el carácter de última ratio del derecho penal.

Por su parte, la Sala II de la Cámara Federal de Casación Penal (según los votos de la Dra. Ángela Ledesma y Alejandro Slokar), se ha encargado de expedirse al respecto 5 .

En el citado fallo del año 2012, en el marco de una causa en la cual se juzgaba a un individuo por el intento de apoderamiento

\footnotetext{
4 Cámara Nacional de Apelaciones en lo Criminal y Correccional, Sala VI, 05/11/2004, causa n² 25.041, "Bargas"; Causa n² 28.348, 15/03/2006 "Gómez"; 20/06/2006, Causa "Bueno" y; 20/06/2006- Causa n ${ }^{0} 31.326$ "Rosich", entre otras.

5 Cámara Federal de Casación Penal, Sala II, causa 15.556, 8/05/12, caratulada "Gerbasi, H. H. s/recurso de casación", registro n 20.751
} 
de dos piezas de carne del interior de un supermercado "Día", la Dra. Ledesma esbozó que en los sistemas inquisitivos como los que mayormente encontramos en la órbita de la justicia federal, en parte de sus horizontes tienen como epicentro a la infracción al orden interno y no en el abordaje del conflicto. Así pues, del fallo se desprende que:

Basta con la mera lesión al ordenamiento para que se articulen de manera automática (y muchas veces irracional) todos los mecanismos burocráticos estatales de persecución penal, sin una finalidad encaminada a ordenar esa conflictividad, gestionarla y finalmente dar soluciones específicas. En sentido opuesto, los sistemas de justicia de bases más democráticas, se fundan en la noción de conflicto, lo cual significa que se privilegia la resolución de los casos mediante parámetros de tolerancia y de no abuso de poder, con una clara atención al caso concreto, a la víctima afectada y al alcance del daño producido. Claramente, si la noción fundamental es el conflicto, su ausencia, o su mínima incidencia en el entramado social, determinan la retracción de los poderes punitivos para abrir camino a otro tipo de respuestas menos violentas.

En el mismo sentido, el Dr. Slokar advirtió que, respecto la irracionalidad del sistema, el mismo:

No ofrece respuestas diferenciadas de acuerdo a la problemática concreta del caso, sino que aplica automáticamente la violencia estatal frente al mero incumplimiento. Esta lógica atenta contra una buena administración de justicia, pues impide que los esfuerzos de jueces, fiscales y funcionarios se oriente a los casos de mayor complejidad y/o trascendencia social (...) hay una discriminación que es estructural del poder punitivo, empero, al mismo tiempo, existe una mecánica que puede llevar a los funcionarios actuantes a extremos tales de perder toda estimativa jurídica, impulsando el proceso y hasta acelerando el juicio para justificar demoras y adoptar decisiones que -prefiero así sospecharlo- deben repugnar a su propia conciencia. 
Para finalizar el presente análisis y no caer en reiteraciones inútiles, nuestra jurisprudencia cuenta con muchos antecedentes que excluyen la tipicidad de los hechos que poseen una ínfima o menor lesividad siempre que se tenga especial consideración en las circunstancias particulares del caso (Cámara Nacional de Apelaciones en lo Criminal y Correccional, Sala V -10/06/2010-Causa n'39.227 "Brizzolaro, Marta Isabel"; in re: causa $n^{\circ} 38.573$-3/3/2010- "Ramos"; Causa no 267 de la Sala de Feria "-28/1/09- "Drogo"; Cámara Nacional de Casación Penal, sala III -8/6/2004-, "Ruiz de la Vega, Carolina"; entre muchos otros).

\section{ii. Perspectiva procesal y los criterios de oportunidad}

Ahora bien, siguiendo el orden de ideas respecto del abordaje de la injerencia del principio de insignificancia en el delito de hurto por cuanto al menor o ínfimo valor de la cosa sustraída, podemos observar la corriente que pregona su fundamento desde una órbita o faz procesalista.

Dichas tesituras presentan la solución como una circunstancia ajena a la tipicidad de la conducta atribuida independientemente del mayor o menor valor de la cosa sustraída y establecen que, a sus efectos, debe acudirse a criterios de oportunidad que den cuenta de la ajenidad de los hechos significantes en la órbita del Derecho Penal.

Antes de adentrarme en la cuestión, huelga hacer mención que en nuestro sistema, la aplicación del principio de oportunidad puede estar íntimamente relacionado con posturas utilitaristas o legitimantes del fin y los límites a la imposición de las penas.

Vale decir que, podremos vincular al principio de oportunidad con finalidades político-criminales utilitarias, ya sea respecto la penalización o despenalización de ciertas conductas o bien con el intento de derivar comportamientos punibles hacia formas de tratamiento de conflicto y soluciones alternativas ajenas al Derecho Penal. 
Siguiendo la tesitura del maestro Maier (2004:835-836), la afirmación del principio de oportunidad resulta un intento por redireccionar la selección según fines concretos, tendientes a racionalizar el sistema. Es por ello, que establece que oportunidad significa:

La posibilidad de que los órganos públicos, a quienes se les encomienda la persecución penal, prescindan de ella, en presencia de la noticia de un hecho punible o, inclusive, frente a la prueba más o menos completa de su perpetración, formal o informalmente, temporal o definitivamente, condicionada $o$ incondicionadamente, por motivos de utilidad social o razones político-criminales.

En nuestro sistema, donde en la mayoría de los códigos rituales se encuentran regulados estos criterios de oportunidad con los cuales cuenta el Estado titular de la acción penal (a cargo del Ministerio Público Fiscal), no debe negarse que dicha herramienta hace las veces de racionalizadora de la selectividad intrínseca con la que cuenta el sistema penal, y que, al darle al Fiscal la potestad de dejar de lado aquellos hechos en donde se contemple innecesaria la aplicación del ius puniendi, se contribuye así a una eficiencia real del sistema, posibilitando excluir los hechos de menor entidad (como los hurtos bagatelares en tratamiento, por ejemplo) y dejar así librado el sistema a efectos de darle tratamiento a aquellos casos que indudablemente requieren la efectiva intervención de la justicia penal (Guariglia, 1993:88).

Dicha herramienta con la que cuenta el titular de la acción penal ha sido receptada en provincias como Río Negro, Mendoza, Neuquén, La Pampa, Chubut y Buenos Aires entre otras, lo cual reduce aún más el margen de actuación en torno a este tipo de delitos.

En relación a ello, por ejemplo, bien reza el Código Procesal Penal bonaerense, no dando margen a interpretación diferente respecto dicho principio que, el Ministerio Público Fiscal podrá archivar las actuaciones respecto de uno o varios de los hechos 
imputados, o de uno o más de los partícipes, en los siguientes supuestos: "1. Cuando la afectación del bien jurídico o el aporte del imputado en el hecho fuera insignificante y siempre que la pena máxima del delito imputado no supere los seis años de prisión [...]". (Código Procesal Penal de Buenos Aires, Ley $13.943,2009$, art. 56 bis)

A su vez, el actual Código Procesal Penal de la Nación (cuya denominación actualmente ha sido modificada a Código Procesal Penal Federal), recepta que:

Los representantes del Ministerio Público Fiscal podrán prescindir total o parcialmente del ejercicio de la acción penal pública o limitarla a alguna de las personas que intervinieron en el hecho en los casos siguientes: a) Si se tratara de un hecho que por su insignificancia no afectara gravemente el interés público [...] (Código Procesal Penal Federal, Ley 27482, 2019, art. 31)

Cabe destacar que, dichas facultades fueron convalidadas por la incorporación del inciso $5^{\circ}$ al artículo 59 del Código Penal, que lo contempla como causal de extinción de la acción penal al legislar la misma ante "la aplicación de un criterio de oportunidad, de conformidad con lo previsto en las leyes procesales correspondientes" (Ley 27.147, 2015, art.59).

Para finalizar con el presente análisis, creeríamos que en el caso en estudio, desconocer la relevancia del Ministerio Público Fiscal en cuanto a la aplicación de criterios de oportunidad y su concreción en cuanto a los criterios especiales de archivo reglados, atentaría contra un adecuado sistema penal en donde se pondría de manifiesto una cierta selectividad delictiva haciendo énfasis en sectores vulnerables y la imposición de su castigo por las sustracciones de objetos de ínfimo valor patrimonial. Asimismo, estimamos que ello haría caso omiso e incurriría en un descuido respecto los delitos más complejos que sí incidan al sistema y contemplen a una afectación mucho más significativa dentro de la sociedad, atendiendo así a criterios político-criminales ante lesiones de una gravedad superior respecto los bienes jurídicos los cuales el legislador pretendió resguardar. 


\section{b. Rechazo del principio de insignificancia como causal de exclusión típica respecto ciertas conductas lesivas}

Culminando con la exposición, resta por desentrañar lo argumentado por la corriente que rechaza la aplicación de estos criterios como excluyentes de la tipicidad en las conductas mencionadas.

Observamos un sector mayoritario de la doctrina, el cual recepta y adhiere a la tesitura entendedora respecto la irrelevancia de la magnitud del valor patrimonial del objeto sustraído como elemento tipificante de la conducta delictiva.

Soler (1970:214) sostuvo oportunamente que en relación con el valor de la cosa, "este delito, está calificado por la ley, como delito contra la propiedad y este derecho es independiente del valor económico de cambio que el bien mismo puede tener". Seguidamente, arguyó que bastará con que "[...] una cosa tenga el carácter de tal, y que esté en el patrimonio de alguien, para que pueda ser objeto de hurto, aun cuando ella carezca de valor para los demás, inclusive para el ladrón [...]".

En consonancia con lo sostenido por Sebastián Soler, también toman postura Creus y Buompadre (2013:430) respecto la cosa y su valor patrimonial. Así pues, para los autores "el valor patrimonial de la cosa no coincide estrictamente con su valor económico, en cuanto éste se entienda como valor de uso o cambio", por lo que "[...] el nulo o ínfimo valor de la cosa en ese sentido no le quita el carácter de objeto de delito" lo cual concuerda con amplia jurisprudencia nacional que se ha expedido al respecto.

En este orden de ideas, parecería ser que para la mayoría de la jurisprudencia en el territorio nacional, fue determinante la afirmación respecto que "todo injusto penal requiere la afectación -sea por vía de lesión o peligro- del bien jurídico. La afectación a aquél existe o no existe, se da o no se da y aún cuando la lesión sea mínima, en alguna medida ha sido vulnerado".

6 Cámara Nacional Criminal y Correccional, Sala de Feria, 23/01/2004, “Álvarez Rodriguez y otros". Sumario SAIJ: FA04060030. Recuperado de: http://www.saij.gob.ar/camara- 
Así pues, en orden a las diferentes posturas adoptadas respecto la insignificancia, la postura mayoritaria que rechaza la no punibilidad de los hurtos bagatelares siempre se ha valido de un precedente, no menos importante claro, que data del año 1986. El mismo posee una antigüedad de treinta y cuatro años, al cual ha sido refutado en alguna oportunidad por fallos de la Cámara Nacional Criminal y Correccional, debido a la vetustez con la cual data el mismo ${ }^{7}$.

El 25 de septiembre de 1986, la Corte Suprema de Justicia de la Nación, se expidió en su leading case "Adami", respecto la improcedencia de defensas en cuanto al principio de insignificancia en los hurtos bagatelares, estableciendo su postura de manera que no dio lugar a interpretación en contrario.

Así fue que el máximo tribunal nacional estableció que:

De la manera que se encuentra legislado el hurto, cualquiera sea la afectación del bien tutelado que resulte como consecuencia del apoderamiento ilegítimo, en tanto no se prevén grados ni límites, hace que la conducta quede comprendida en el referido artículo 162. La insignificancia solo puede jugar cuando es tal que lleva a despojar a la cosa de ese carácter. Es que no se atiende a la entidad de la lesión patrimonial, sino a la violación al derecho de

nacional-apelaciones-criminal-correccional-nacional-ciudad-autonoma-buenos-aires-alvarezrodriguez-tabare-otros--fa04060030-2004-01-23/123456789-030-0604-0ots-eupmocsollaf?

7 Al respecto, en el voto disidente de la Causa "Gerban" de la Sala VII de la Cámara Nacional Criminal y Correccional que data del 31/03/2009, el Dr. Mauro Divito esbozó que la Corte adoptó la postura con el voto de la mayoría conformada por los jueces Caballero, Belluscio y Fayt, pero que sin perjuicio de ello, "la conformación del alto tribunal ha variado, a tal punto que hoy en día queda solamente uno de los jueces que suscribieron la opinión mayoritaria en "Adami" (el juez Fayt), y que uno de sus miembros (el juez Zaffaroni) es quien, en esa misma causa, había emitido el voto principal en la sentencia de segunda instancia que finalmente fue revocada. También cabe apuntar que la Corte Suprema -en su actual composición ha reconocido que el derecho penal debe funcionar como la ultima ratio del ordenamiento jurídico (cfr. considerando $6^{\circ}$ del voto de la mayoría -jueces Lorenzetti, Fayt, Maqueda y Zaffaroni- en el fallo "Acosta, Alejandro Esteban", de fecha 23/4/2008) y es sabido que, conforme a dicho criterio, el poder penal del Estado debe emplearse solamente frente a los ataques graves contra bienes jurídicos, lo que permite excluir del ámbito de lo punible aquellas conductas que los afectan en forma mínima (cfr., en este sentido, Marum, ob. cit., p. 37). 
propiedad, independientemente del mayor o menor valor de la cosa, aspecto que es relevante sólo a los fines de graduar la pena (CSJN, Fallos 308:1976, 25/09/1986).

Sentada la postura del tribunal cimero en el año 1986, innumerables judicaturas de primera instancia y superiores se han expedido en el mismo sentido.

En ellas, el menor o mayor valor de la cosa sustraída no es considerado como elemento indispensable para valorar la tipicidad o atipicidad de la conducta, por lo que la lesión del derecho a la propiedad subsiste de cualquier forma, entendida ella en el sentido amplio asignado por la Carta Magna nacional la cual arribaría a la situación que dicho principio deba ser merituado por el Juez al momento de graduar la pena a imponer en el caso concreto ${ }^{8}$.

\section{Conclusiones y toma de postura}

Llegando al final de este trabajo, se intentó abordar acabadamente el problema desde las corrientes doctrinarias más significantes existentes a la actualidad, lo cual, sin perjuicio de ello, estimo que las aguas se encontrarán divididas hasta tanto no se establezcan legislativamente, al menos, ciertos parámetros que permitan su delimitación.

Resulta a todas luces visible la problemática suscitada en los tribunales nacionales y provinciales respecto de las causas que se instruyen y tramitan, las cuales insume un mayor gasto público el mero hecho de sostener su tramitación que el bien jurídico (patrimonial) en sí que se intenta o intentó dañar, por lo que resulta de ardua tarea arribar a una solución armónica con la letra de la ley y los principios rectores del Derecho Penal que imperan en la materia.

8 Cám. Nac. Crim. y Corr., sala VI, 1/3/2007 - Rosich, Eric. A.; C. Nac. Crim. y Corr., sala I, 11/12/2006 - Casafú, Omar D.; C. Nac. Crim. y Corr., sala VII, 10/03/2011 - B., D. A., ídem causa nro. 27.815 - Castaño, Miguel A., rta. 06/06/05., ídem causa nro. 29.243 - Gil, Marcelo, rta. 26/05/06, TCPBA, Sala IV, causa N 55.114, D.L.E. S/ recurso de casación, 14/05/2013. 
Vimos a su vez cómo el Código Penal Argentino en su redacción, vuelve a generar un cortocircuito, culpa de la ambigüedad (o quizás voluntad del legislador...) con la cual cuenta la figura penal en estudio y más aún el tipo de bien jurídico protegido en estos casos.

Sin perjuicio de entender de manera sólida los argumentos vertidos por la doctrina al respecto, se pueden observar dos grandes problemas que hacen de difícil solución ante el suceso de conductas análogas a la hora de ingresar al sistema judicial:

En primer lugar, se logró advertir que estamos en presencia de una vaguedad y una ambigüedad terminológica que muchas veces se enfrenta a la dificultad que hace que no se pueda arribar a una solución con claridad, visto que resulta de su lectura una falta de precisión que no permite arribar a la conclusión con seguridad respecto de cuándo estamos ante algo insignificante, o cuándo el objeto resulta tan inocuo al patrimonio del sujeto pasivo de la conducta delictiva lo cual lo hace insignificante.

Respecto a ello, estimo adecuada o prima facie válida, la idea respecto que un análisis de la afectación al bien jurídico "propiedad" (con el alcance brevemente mencionado a lo largo de este trabajo), no puede hacerse en forma abstracta, sino que tomando como punto de partida para su análisis la teoría del delito y la afectación del bien jurídico como límite a la tipicidad de una conducta, de modo tal que una lesión insignificante, pueda resultar atípica por no poseer entidad suficiente para requerir la intervención estatal y dar comienzo a su ius puniendi. Es por ello también que como se explicara con anterioridad, no debe perderse de vista el principio general de ultima ratio del Derecho Penal, por lo que a la hora de realizar un estudio dogmático y típico de la conducta imputada debe hacerse en un todo conjunto, y no de manera aislada.

Por otro lado, advierto también un segundo problema. Tornar aplicable la teoría de la insignificancia o tomar como punto de partida para el tratamiento de las conductas presuntamente ilícitas abordadas, teorías como la de la tipicidad y atipicidad 
conglobante brevemente reseñada al comienzo del trabajo, llevarían a una suerte de confusión entre la función de los jueces a cargo de aplicar las normas y el encargado de diseñar las políticas criminales, por lo que advertiría un nuevo cortocircuito, ni más ni menos, entre dos poderes como lo son el Legislativo y el Judicial. Ello así, toda vez que el abordaje de las conductas, prima facie reprochables por parte del juez, lo conduciría a ejercer tareas que no le son propias, por estarle vedado a éste y estar asignadas al Congreso de la Nación.

Como ya fuera desarrollado, sin perjuicio de tornarse de aplicación armónica con la ley fondal la existencia y utilización de criterios de oportunidad en cabeza del titular de la acción penal, advierto que el avance de las causas y las conductas reprochables de esta magnitud, hacen que la reacción penal juegue un papel desproporcionado, más aún cuando estamos en presencia de lesiones mínimas o insignificantes, dado que ello no conmueve el manto de tipicidad de la conducta enrostrada al sujeto activo del delito.

Es por ello que en el presente caso, de no haberse acudido a criterios de oportunidad o archivo especial durante la etapa investigativa, podría acudirse a métodos alternativos de resolución de conflictos, los cuales al fin y al cabo, han sido establecidos por el legislador a los efectos de evitar la estigmatización que conlleva el sometimiento del imputado a procesos de esta clase y la amenaza de una eventual imposición de una pena, frente a la posibilidad de arribar a una solución del conflicto que intente satisfacer los intereses de las víctimas y permita así intentar en los mismos la asimilación de ciertas pautas de conducta de una manera no represiva, tales como podrían ser los institutos de la mediación penal o la suspensión del juicio a prueba.

\section{Bibliografía}

Creus, Carlos (1998) Derecho Penal. Parte Especial. Tomo I, 6ta. Edición actualizada y ampliada. Editorial Astrea. Buenos Aires. 
Creus, Carlos y Jorge Enrique Buompadre (2013) Derecho Penal, Parte Especial. Tomo I, 7ma edición. Editorial Astrea. Buenos Aires.

Donna, Edgardo Alberto (2007) Derecho Penal: Parte Especial. Tomo II. Editorial Rubinzal Culzoni. Buenos Aires.

Guariglia, Fabricio (1993) "Facultades discrecionales del ministerio público e investigación preparatoria: el principio de oportunidad" en El Ministerio Público en el proceso Penal. Editorial Ad Hoc. Buenos Aires.

Maier, Julio Bernardo José (2004). Derecho Procesal Penal. Fundamentos. Tomo I 2da. Edición. Editorial Del Puerto. Buenos Aires.

Roxin, Claus (1997). Derecho Penal. Parte General. Tomo I. Editorial Civitas. Madrid.

Soler, Sebastián (1970) Derecho Penal argentino. Tomo IV. Editorial Tea. Buenos Aires.

Tribunal de Casación Penal de Buenos Aires (2013) D.L.E. S/ recurso de casación. En línea en: www.saij.gob.ar/camaranacional-casacion-penal-federal-ciudad-autonoma-buenosaires-otro-recurso-casacion-fa13261005-2013-02-21/123456789500-1623-1ots-eupmocsollaf

- (2017) Tello, Daniel David s/ recurso de casación. En línea en: www.scba.gov.ar/includes/descarga.asp?id=37254\&n=Ver $\% 20$ Sentencia \%20(78444).pdf

Tribunal Oral en lo Criminal Nro. 1 de Necochea (2014) Olivares Mario Alberto s/ robo en agravado por efracción, Buenos Aires, Argentina.

Zaffaroni, Raúl Eugenio (1981) Tratado de Derecho Penal. Parte General. Tomo III. Editorial Ediar. Buenos Aires.

Zaffaroni, Eugenio Raúl, Alagia, Alejandro y Slokar, Alejandro (2014) Manual de Derecho penal: Parte General. Editorial Ediar. Buenos Aires| 